\title{
Nucleon-Nucleon Interactions and Observables
}

\author{
Wayne N. Polyzou \\ Department of Physics and Astronomy, The University of Iowa, Iowa City, IA 52242
}

(August 13, 2018)

\begin{abstract}
A class of nucleon-nucleon interactions which are exactly phase equivalent to a given realistic nucleon-nucleon interaction are exhibited. These interaction have the property that the RMS radius of the deuteron can be made arbitrarily large without changing any of the scattering or bound-state observables. With this construction it is possible to find realistic interactions that do not obey the linear relation between the RMS radius and the triplet scattering length observed by Klarsfeld et. al. [1]. The interpretation of these examples is discussed.
\end{abstract}

${ }^{0}$ P.A.C.S. 13.175 c, 21.60 


\section{INTRODUCTION}

In 1986 Klarsfeld, Martorell, Oteo, Nishimura, and Sprung [1] observed an empirical linear relation between the triplet scattering length and the deuteron RMS radius in a large class of realistic nucleon-nucleon interactions. In this paper I exhibit sequences of realistic nucleon-nucleon interactions that have identical S-matrix elements, identical bound-state observables, and arbitrarily large deuteron RMS radius. In addition, for each interaction there is an exchange current that leaves all electromagnetic observables unchanged.

The construction of the model interactions is based on two observations. The first is that unitary transformations that are compact perturbations of the identity leave all scattering and bound state observables unchanged. The second that $r^{2}$ is an unbounded operator. Using these two observations it is possible to construct model interactions with the desired properties. The resulting model interactions differ from the given initial nucleon-nucleon interaction on a two-dimensional subspace of the Hilbert space.

The key result of this paper is the following theorem:

Theorem: Let $V:=V_{0}$ be a realistic nucleon-nucleon interaction with Hamiltonian $H=$ $H_{0}=K+V_{0}$. Let $r_{0}$ be the deuteron RMS radius in this model. There exist an infinite sequence of interactions $\left\{V_{m}\right\}_{m=1}^{\infty}$ and two-body Hamiltonians $H_{m}=K+V_{m}$ with the properties

1. Each $H_{m}$ has the same spectrum and scattering observables as $H_{0}$.

2. If $\left|\psi_{m}\right\rangle$ is the deuteron eigenstate of $H_{m}$ then

$$
\left\langle r^{2}>_{m}:=\left\langle\psi_{m}\left|r^{2}\right| \psi_{m}\right\rangle \geq m r_{0}^{2} .\right.
$$

\section{PROOF OF THE THEOREM}

In all that follows I assume that all operators are considered to have domain and range in the relative motion Hilbert space $\mathcal{H}_{\text {rel }}$ defined by:

$$
\mathcal{H}=\mathcal{L}^{2}\left(R^{3}, d^{3} p\right) \otimes \mathcal{H}_{\text {rel }}
$$

To prove the theorem I first exhibit a class of unitary transformations that preserve all bound state and scattering observables. To do this let $|\chi\rangle$ be any unit normalized vector and define

$$
W:=I+\frac{2 i}{1-i}|\chi\rangle\langle\chi| .
$$

Direct computation shows that

$$
W^{\dagger} W=I \text {. }
$$

Next note that if [2] 


$$
\lim _{t \rightarrow \pm \infty} \|(W-I) e^{-i K t}|\phi\rangle \|=0
$$

for every vector $|\phi\rangle \in \mathcal{H}_{\text {rel }}$ and both time limits then it follows that

$$
\begin{gathered}
0=\lim _{t \rightarrow \pm \infty} \| e^{i H t}(W-I) e^{-i K t}|\phi\rangle \|= \\
\lim _{t \rightarrow \pm \infty} \|\left(W e^{i W^{\dagger} H W t}-e^{i H t}\right) e^{-i K t}|\phi\rangle \|= \\
\|\left[W \Omega_{ \pm}\left(W^{\dagger} H W\right)-\Omega_{ \pm}(H)\right]|\phi\rangle \| .
\end{gathered}
$$

With $H^{\prime}$ defined by

$$
H^{\prime}:=W^{\dagger} H W
$$

equation (5) implies the Møller wave operators for $H$ and $H^{\prime}$ are related by

$$
\Omega_{ \pm}(H)=W \Omega_{ \pm}\left(H^{\prime}\right)
$$

which is valid for both asymptotic conditions. It follows that the scattering operators $S$ and $S^{\prime}$ for $H$ and $H^{\prime}$ are identical:

$$
\begin{gathered}
S:=\Omega_{+}^{\dagger}(H) \Omega_{-}(H)= \\
\Omega_{+}^{\dagger}\left(H^{\prime}\right) W^{\dagger} W \Omega_{-}\left(H^{\prime}\right)=\Omega_{+}^{\dagger}\left(H^{\prime}\right) \Omega_{-}\left(H^{\prime}\right)=S^{\prime} .
\end{gathered}
$$

Note that the free relative kinetic energy $K$ is not transformed in the wave operator.

This shows that any unitary transformation $W$ satisfying (5) will preserve the spectrum and all scattering observables. The resulting transformed Hamiltonian has the form

$$
H^{\prime}=H+\frac{2 i}{1-i}|\chi\rangle\left\langle\chi\left|H-\frac{2 i}{1+i} H\right| \chi\right\rangle\langle\chi|+2| \chi\rangle\langle\chi|H| \chi\rangle\langle\chi|
$$

which differs from $H$ only on the two dimensional subspace spanned by $|\chi\rangle$ and $H|\chi\rangle$.

To establish the desired equivalence it is sufficient to prove (5) for $W$ of the form (3). This is equivalent to establishing

$$
\lim _{t \rightarrow \pm \infty}\left|\left\langle\chi\left|e^{-i K t}\right| \psi\right\rangle\right|=0
$$

Inserting intermediate eigenstates of $K$, realizing that $K$ has absolutely continuous spectrum, gives:

$$
\begin{gathered}
\lim _{t \rightarrow \pm \infty}\left|\int_{0}^{\infty} e^{-i k \cdot t} \int d x\langle\chi \mid k, x\rangle\langle k, x \mid \psi\rangle\right|= \\
\lim _{t \rightarrow \pm \infty}\left|\int_{0}^{\infty} d k f(k) e^{-i k \cdot t}\right|=0 .
\end{gathered}
$$


Since

$$
f(k):=\int d x\langle\chi \mid k, x\rangle\langle k, x \mid \psi\rangle
$$

is a product of square integrable functions of $k$ it is an $L^{1}$ function of $k$ after integrating out the other observables $x$. The vanishing of (12) is a consequence of the Riemann-Lebesgue lemma [3].

This establishes that all Hamiltonians $H^{\prime}$ of the form (10) have the same bound state and scattering observables as the initial Hamiltonian $H$.

To complete the proof of the theorem I show that it possible, by a careful choice of the vectors $|\chi\rangle$, to make the deuteron RMS radius as large as desired. To begin the construction first note that the deuteron RMS radius for the $H^{\prime}$ Hamiltonian is

$$
\begin{array}{r}
\left\langle r^{2}\right\rangle^{\prime}:=\left\langle\psi^{\prime}\left|r^{2}\right| \psi^{\prime}\right\rangle=\left\langle\psi\left|W r^{2} W^{\dagger}\right| \psi\right\rangle= \\
\left\langle\psi\left|\left(1+\frac{2 i}{1-i}|\chi\rangle\langle\chi|\right) r^{2}\left(1-\frac{2 i}{1+i}|\chi\rangle\langle\chi|\right)\right| \psi\right\rangle .
\end{array}
$$

Choose $|\chi\rangle$ so it satisfies

$$
\left\langle\chi\left|r^{2}\right| \psi\right\rangle=\left\langle\psi\left|r^{2}\right| \chi\right\rangle=0
$$

and

$$
\langle\chi \mid \psi\rangle \neq 0
$$

With this choice the cross terms in (14) do not contribute which gives the following expression for the deuteron RMS radius:

$$
\left\langle r^{2}\right\rangle^{\prime}=\left\langle\psi\left|r^{2}\right| \psi\right\rangle+2|\langle\psi \mid \chi\rangle|^{2}\left\langle\chi\left|r^{2}\right| \chi\right\rangle
$$

The theorem is proved by exhibiting a sequence of $\left|\chi_{m}\right\rangle$ satisfying (15-16) with the property that $\left|\left\langle\psi \mid \chi_{m}\right\rangle\right|$ is bounded from below, and $\left\langle\chi_{m}\left|r^{2}\right| \chi_{m}\right\rangle$ is arbitrarily large.

To construct such a sequence of vectors let

$$
\left|\xi_{0}\right\rangle=N r^{2}|\psi\rangle
$$

where $N$ is a constant chosen to make this vector normalized to unity. Let $\left\{\left|\xi_{m}\right\rangle\right\}_{n=0}^{\infty}$ any complete orthonormal basis in the domain of $r^{2}$ with $\left|\xi_{0}\right\rangle$ as the first vector and $\left|\xi_{1}\right\rangle$ satisfying

$$
\left\langle\xi_{1} \mid \psi\right\rangle=c=c^{*}>0
$$

Note the following properties:

$$
\begin{gathered}
\lim _{n \rightarrow \infty}\left\langle\xi_{n} \mid \psi\right\rangle=0 \\
\lim _{n \rightarrow \infty}\left\langle\xi_{n}\left|r^{2}\right| \xi_{1}\right\rangle=0
\end{gathered}
$$


and for each finite $R>0$ there is an $n$ such that

$$
\left\langle\xi_{n}\left|r^{2}\right| \xi_{n}\right\rangle>R
$$

The first two properties are required for $|\phi\rangle$ and $r^{2}|\phi\rangle$ be normalizable. If the last property is a mathematical form of the statement that $r^{2}$ is unbounded.

What these properties mean is given any arbitrarily small $\epsilon>0$ and any finite $R>0$ it is possible to find an $n=n(\epsilon, R)$ such that

$$
\begin{gathered}
\left|\left\langle\xi_{n} \mid \psi\right\rangle\right|<\epsilon \\
\left|\left\langle\xi_{n}\left|r^{2}\right| \xi_{1}\right\rangle\right|<\epsilon r_{0}^{2} \\
\left|\left\langle\xi_{n}\left|r^{2}\right| \xi_{n}\right\rangle\right|>R .
\end{gathered}
$$

Let $m$ be a given integer, choose $n$ so $\epsilon=.01 c$, and $R$ satisfying

$$
R>\frac{2(m-1) r_{0}^{2}}{(.99 c)^{2}}+.02 c r_{0}^{2}-r_{1}^{2}
$$

where

$$
r_{1}^{2}:=\left\langle\xi_{1}\left|r^{2}\right| \xi_{1}\right\rangle
$$

For this choice of $m$ define

$$
\left|\chi_{m}\right\rangle:=\frac{1}{\sqrt{2}}\left(\left|\xi_{1}\right\rangle+\left|\xi_{n}\right\rangle\right) .
$$

By construction

$$
\begin{gathered}
\left\langle\xi_{n} \mid \psi\right\rangle=\epsilon_{m} e^{i \phi_{m}} \quad \epsilon_{m} \leq \epsilon \\
\left\langle\xi_{n}\left|r^{2}\right| \xi_{1}\right\rangle=r_{0}^{2} \epsilon_{m}^{\prime} e^{i \phi_{m}^{\prime}} \quad \epsilon_{m}^{\prime} \leq \epsilon .
\end{gathered}
$$

It follows that

$$
\begin{gathered}
\left\langle\psi_{m}\left|r^{2}\right| \psi_{m}\right\rangle= \\
r_{0}^{2}+2\left|\left\langle\psi \mid \chi_{m}\right\rangle\right|^{2}\left\langle\chi_{m}\left|r^{2}\right| \chi_{m}\right\rangle= \\
r_{0}^{2}+\frac{1}{2}\left|c^{2}+2 \epsilon_{m} \cos \left(\phi_{m}\right)+\epsilon_{m}^{2}\right|\left[r_{1}^{2}+2 r_{0}^{2} \epsilon_{m}^{\prime} \cos \left(\phi_{m}^{\prime}\right)+\left\langle\xi_{n}\left|r^{2}\right| \xi_{n}\right\rangle\right] .
\end{gathered}
$$

The relations (19), (23-26), and (29-30) imply

$$
\left|c^{2}+2 \epsilon_{m} \cos \left(\phi_{m}\right)+\epsilon_{m}^{2}\right|>|c-\epsilon|^{2}=(.99 c)^{2}
$$


and

$$
\begin{aligned}
& {\left[r_{1}^{2}+2 r_{0}^{2} \epsilon_{m}^{\prime} \cos \left(\phi_{m}^{\prime}\right)+\left\langle\xi_{n}\left|r^{2}\right| \xi_{n}\right\rangle\right] \geq} \\
& r_{1}^{2}-2 r_{0}^{2} \epsilon+R=r_{1}^{2}-.02 c r_{0}^{2}+R .
\end{aligned}
$$

Putting these together gives

$$
\begin{gathered}
\left\langle\psi_{m}\left|r^{2}\right| \psi_{m}\right\rangle \geq \\
r_{0}^{2}+\frac{1}{2}(.99 c)^{2}\left(r_{1}^{2}-.02 c r_{0}^{2}+R\right) .
\end{gathered}
$$

Choosing $R$ satisfying (26) gives

$$
\left\langle\psi_{m}\left|r^{2}\right| \psi_{m}\right\rangle \geq m r_{0}^{2}
$$

which completes the proof of the theorem.

The relevant interactions have the general form

$$
\begin{gathered}
V_{m}= \\
V+\frac{2 i}{1-i}\left|\chi_{m}\right\rangle\left\langle\chi_{m}\left|H-\frac{2 i}{1+i} H\right| \chi_{m}\right\rangle\left\langle\chi_{m}|+2| \chi_{m}\right\rangle\left\langle\chi_{m}|H| \chi_{m}\right\rangle\left\langle\chi_{m}\right| .
\end{gathered}
$$

Since each of these interactions all have the same scattering operator they all have the same triplet scattering length. The deuteron RMS radius can be made as large as desired by choosing $m$ sufficiently large. Note that the radius can be adjusted continuously from $r_{m}$ to $r_{m+1}$ by interpolating between $\left|\chi_{m}\right\rangle$ and $\left|\chi_{m+1}\right\rangle$ using

$$
|\chi\rangle \rightarrow \frac{\lambda\left|\chi_{m}\right\rangle+(1-\lambda)\left|\chi_{m+1}\right\rangle}{\sqrt{2 \lambda^{2}-2 \lambda+1}} .
$$

\section{INTERPRETATION}

The interactions constructed in this paper show by explicit example that an interaction fit to bound state and scattering data need not have any relation between triplet scattering length as deuteron RMS radius. The models constructed all have a separable nonlocality that acts on a two-dimensional subspace.

This observation is related to the fact that the RMS radius is not an observable. The RMS radius should be distinguished from the charge radius which is a scattering observable. In the models constructed in this paper the hadronic current must also be transformed with the same unitary transformation. If this is done the electromagnetic observables remain

unchanged, however this is achieved by introducing exchange currents. Specifically given the hadronic current operator in the 0 representation, the current in the $m$-representation is related by 


$$
J_{m}^{\mu}(x)=W_{m}^{\dagger} J_{0}^{\mu}(x) W_{m}
$$

or

$$
J_{0}^{\mu}(x)=W_{m} J^{\mu}(x) W_{m}^{\dagger} .
$$

The two-body parts of the unitary operators $W_{m}$ generate exchange current contributions.

The result of the construction in this paper is a collection of Hamiltonians $H_{m}$ and currents $J_{m}^{\mu}(x)$ that give the same predictions for bound state, scattering, and electromagnetic observables. A preferred interaction can be identified by requiring that there are no exchange currents in the charge density. This view is certainly supported by historical prejudice and is the basis of most non-relativistic phenomenology. This assumption is consistent with non-relativistic quantum mechanics but it is unfortunately inconsistent with Poincaré invariance, which requires that the charge density satisfy [4]:

$$
\begin{gathered}
{[H, \rho(0)]+i \sum_{l=1}^{3}\left[P^{l},\left[K^{l}, \rho(0)\right]\right]=0} \\
{[\vec{J}, \rho(0)]=0} \\
{\left[K^{l},\left[K^{l}, \rho(0)\right]\right]+\rho(0)=0}
\end{gathered}
$$

where $H, \vec{P}, \vec{J}, \vec{K}$ are the Poincaré generators. These relations cannot be satisfied by an impulse charge density in a model with interactions. This problem cannot be defined away by a clever unitary transformation.

In order to understand why a perfectly good Hermitian operator should not be considered an observable it is useful to consider the difference between any two of the representations corresponding to $m^{\prime} \neq m$. Both models are fit that same experimental data. They differ by a unitary transformation, $W_{m}^{\dagger} W_{m^{\prime}}$, which looks like the identity when the particles are far apart, but has some correlations when they are close together. If we consider this unitary transformation as a change of coordinates then both coordinate systems look the same when considering the behavior of asymptotically separated free particles. In both models the free Hamiltonian is has the same form (as it is used to compute the $S$-matrix). The $m$ representation appears to have a coordinate system with short range correlations relative to the $m^{\prime}$ while the $m^{\prime}$ representation appears to have a coordinate system with short range correlations relative to the $m$ system. The models cannot be distinguished based on how well they fit bound state and scattering observables.

The problem is that most experiments only measure asymptotic particles. There are clearly some limitations to this picture. Particles are not measured at infinite distances from the interaction region. Models having a deuteron with a macroscopic RMS radius would require a bizarre reinterpretation of physics; on the other hand without a probe that explicitly couples to the strong RMS radius, it is impossible to find an experimental basis for preferring a model with a specific RMS radius. One consequence of this theorem is that it always possible to introduce non-localities that will make the RMS radius identical to the charge radius. 
The main message in this purely academic exercise is that in the absence of an interaction that couples directly to the RMS radius, the RMS radius is not a true quantum mechanical observable. It is a representation dependent quantity where the "correct" choice of representation cannot be determined by experiment. More practical considerations related to interpreting various radii as observables are discussed in a recent preprint of Friar, Martoerll, and Sprung [5]. Derivations of nucleon-nucleon interactions from field theory models also make specific "approximations" which correspond to different representations in the above sense. These considerations make it clear that there is no preferred NN-interaction. It is fortuitous that the many-body physics seems to be relatively insensitive to specific choices of realistic interactions.

\section{ACKNOWLEDGEMENTS}

The author is grateful to Fritz Coester, Sid Coon, and Jim Friar for comments that

contributed materially to this work. This work was supported by the Department of Energy, Nuclear Physics Division, under contract DE-FG02-86ER40286. 


\section{REFERENCES}

[1] S. Klarsfeld, J. Martorell, J. Oteo, M. Nishimura, and D. Sprung, Nucl. Phys. A 456,373(1986).

[2] F. Coester and W. N. Polyzou, Phys. Rev. D 26,1836(1982).

[3] Y. Katznelson, An Introduction to Harmonic Analysis, (Dover, New York 1976).

[4] W. N. Polyzou, Phys. Rev. D 32,2216(1985).

[5] J. Friar, J. Martorell, and D. Sprung, preprint - nucl-th/9707016, July 1997. 